\title{
Medienbildung in der ersten Phase der Lehrkräftebildung und die Bedeutung des medialen Habitus von Lehramtsstudierenden
}

\section{Sue-Ann Bäsler}

\section{Zusammenfassung}

Die medienpädagogische Ausbildung und eine positiv erlebte Medienbildung bei angehenden Lehrkräften sind wichtige Voraussetzungen für eine gelungene Medienbildung in Schule und Unterricht. Der Beitrag setzt sich mit den medienbezogenen Vorstellungen von Lehramtsstudierenden in Hinblick auf das Bildungspotenzial von Medien auf der einen und die medienpädagogische Ausbildung in der ersten Phase der Lehrkräftebildung auf der anderen Seite auseinander. Im Beitrag werden Ergebnisse aus einer qualitativ angelegten Forschungsstudie vorgestellt. Ziel der Studie ist die Benennung von Implikationen für die Medienbildung in der Lehrkräftebildung. Das Konzept des medialen Habitus wird dabei als Erklärungsmoment für medienbezogene Vorstellungen herangezogen, empirisch fundiert und weiterentwickelt.

\section{Schlüsselwörter}

Medienbildung • Digitale Bildung • Lehrkräftebildung • Medienpädagogische Kompetenz $\bullet$ Medialer Habitus $\bullet$ Neue Medien in Schule und Unterricht

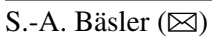

Cornelsen Verlag, Berlin, Deutschland

E-Mail: sue-ann.baesler@gmx.de 


\section{$1 \quad$ Medienbildung als Antwort auf gesellschaftliche Herausforderungen?}

2020 war das Jahr, in dem Bildung zu einer noch nie da gewesenen Herausforderung vieler Menschen wurde. Tausende waren gezwungen, in die Rolle von Lehrkräften zu schlüpfen, um Kindern und Jugendlichen über Monate hinweg ein angemessenes Homeschooling zu bieten. Auf der anderen Seite standen Lehrkräfte, die in dieser Situation versuchten ihren Kernaufgaben - Erziehen und Unterrichten - gerecht zu werden. Mediale Hilfsmittel waren fortan nicht mehr Gegenstand einer fortwährenden Diskussion um die Digitalisierung, sondern greifbare und höchst notwendige Realität. Das Verschwimmen von formellen, informellen, institutionellen und nichtinformellen Lern- und Bildungsgelegenheiten zeichnet sich als nur ein Merkmal dieser Realität aus. Der Begriff der „Medienbildung“ wurde in diesen Zeiten der besonderen bildungsbezogenen Herausforderungen erneut ins öffentliche Bewusstsein gerückt. Medienbildung ist eine wichtige Investition in den Menschen, um Teilhabe, Persönlichkeitsentwicklung und individuelle Lebensgestaltung zu ermöglichen, und kann in allen Bereichen institutionalisierter Bildung stattfinden (Eickelmann et al. 2013). Medienpädagogische Kompetenz (Blömeke 2003) von Lehrkräften ist als Gelingensfaktor für die Medienbildung von Schulkindern und Auszubildenden zu betrachten (Kammerl und Ostermann 2010). Neben einer medienpädagogischen Ausbildung für Lehrkräfte kann auch deren Einstellung zu Medien im Bildungskontext einen Erfolgsfaktor oder ein Hemmnis für erfolgreiche Medienbildung in Schule und Unterricht darstellen (vgl. zum Beispiel Biermann 2009; Biermann 2013; Mayrberger 2012; Scheuble et al. 2014; Lorenz 2018). Als mögliche Erklärung für eine weniger erfolgreiche Medienbildung wird das Konzept des medialen Habitus nach Kommer und Biermann (2012) von Lehrkräften angeführt.

In einer qualitativ angelegten Studie geht die Autorin der Fragestellung nach, ob und inwiefern die universitäre Lehrkräftebildung auf den medialen Habitus von Lehramtsstudierenden einwirkt und inwiefern sich medienpädagogische Ausbildung und medialer Habitus von Lehramtsstudierenden gegenseitig bedingen (Bäsler 2019). Als Ergebnis der Studie werden Implikationen für eine Verbesserung der Medienbildung und medienpädagogischen Ausbildung in der Lehrkräftebildung benannt. In diesem Beitrag werden Auszüge aus den Ergebnissen und deren Genese vorgestellt und in den Kontext aktueller bildungspolitischer Entwicklungen gerückt.

Bevor auf die anfangs erwähnte Forschungsarbeit eingegangen wird, folgt zunächst eine Einführung der Begriffe Medienbildung und medialer Habitus im Kontext der Lehrkräftebildung. 


\section{Begriffsbestimmung Medienbildung und Medialer Habitus}

\subsection{Medienbildung und medienpädagogische Ausbildung}

Tulodziecki sieht die Verwendung des Begriffes Medienbildung insofern problematisch, als unter ihm sowohl ein Prozess als auch ein wünschenswerter Zustand beschrieben wird (Tulodziecki 2011). Medienbildung findet sowohl in informellen als auch in formellen Bildungsräumen statt. Eickelmann et al. (2013) stellen heraus, dass sich Medienbildungsangebote an Entwicklungsaufgaben und -herausforderungen des Individuums orientieren müssen, zum Beispiel der Identitätsfindung. Ein Beispiel für institutionalisierte Medienbildung beschreibt das Basiscurriculum Sprachbildung und Medienbildung (LISUM 2015), welches vom Landesinstitut für Schule und Medien Berlin-Brandenburg für die Bundesländer Berlin und Brandenburg für allgemeinbildende Schulen erarbeitet wurde. Medienbildung wird darin als Querschnittsaufgabe schulischer Bildung betrachtet. Nach Niesyto (2012) geht es im Ansatz von Medienbildung als Querschnittsthema nicht „um die Vermittlung eines festen Kanons von Verfügungswissen, sondern um die Bewusstmachung der grundlegenden Medialität von Bildungsprozessen sowie um die Befähigung, situativ und zielgruppenspezifisch medienpädagogische Arrangements entwickeln und zwischen unterschiedlichen Medienkulturen vermitteln zu können“ (ebenda, S. 62 f.). Für Spanhel (2011) enthält der Begriff Medienbildung ,einen Erfolg versprechenden Ansatz in der Diskussion um eine Theorie der Medienpädagogik“ (Spanhel 2011, S. 95 f.). Begriffe stehen für Theorien; demnach steht hinter Medienbildung eine Bildungstheorie. Mit Begriffen sind Handlungsmandate verbunden: Medienbildung impliziert die anthropologische Bedeutung von Medialität für den menschlichen Bildungsprozess zu erkennen und dementsprechend die Bildungsprogramme in unserer mediatisierten Gesellschaft umzugestalten (ebenda, S. 96 ff.). Jörissen und Marotzki (2009) führten als Weiterentwicklung von Marotzkis Entwurf einer strukturalen Bildungstheorie (Marotzki 1990) den Begriff der strukturalen Medienbildung ein. Die Autoren unterscheiden zwischen Lern- und Bildungsprozessen: Lernen und Lernprozesse sind das Bestimmte, also das Gelernte oder Gewusste, und Bildung und Bildungsprozesse sind das Unbestimmte, also der flexible Umgang mit dem Gelernten (Jörissen und Marotzki 2009, S. 21 ff.). Von [Medien-]Bildungsprozessen ist demnach dann zu sprechen, wenn Lernprozesse stattfinden, die sich auf die Veränderung von Ordnungsschemata und Erfahrungsmustern beziehen: [Medien-]Bildungsprozesse verändern das Repertoire an Konzeptionsmöglichkeiten von Welt- und Selbstverhältnissen (ebenda). Die Autorin des vorliegenden 
Beitrags lehnt sich in ihrer Verwendung des Medienbildungsbegriffs an den Prozessbegriff an und definiert diesen im Kontext des zu untersuchenden Forschungsfeldes Lehrkräftebildung als Bildungsprozess unter der Berücksichtigung von Medien sowohl als Bildungsinhalt als auch als Instrument zur Ausgestaltung dieses Prozesses.

Mit medienpädagogischer Kompetenz geht eine Theorie der Kompetenz einher, die auf Handlungen abzielt. Das Modell medienpädagogischer Kompetenz nach Blömeke (2003), welcher für die hier vorgestellten Forschungsergebnisse handlungsleitend war, orientiert sich an den Hauptaufgaben von Lehrkräften, dem Unterrichten und Erziehen: Gespiegelt auf das Kompetenzmodell bilden zwei Dimensionen den Kern des Modells und die Kernaufgaben, die damit für Lehrkräfte verbunden sind: Mediendidaktik und Medienerziehung (ebenda, S. 20). Des Weiteren gehören zu medienpädagogischer Kompetenz Bedingungen, die die Lehrkraft erfüllen muss, wie zum Beispiel die eigene Medienkompetenz, weitere Rahmenbedingungen, auf die eingegangen werden muss, wie die Lernvoraussetzungen, die Schüler*innen mitbringen, sowie institutionelle Rahmenbedingungen (ebenda).

\subsection{Medialer Habitus}

Der Begriff Habitus nach bourdieuschem Verständnis meint das grundlegende Selbst- und Weltverständnis eines Individuums, das durch den sozialen Raum und die damit verbundenen Lebensbedingungen, unter denen es aufwächst, geprägt wird. Der so entwickelte Habitus gibt vor, wie der soziale Raum vom Individuum bewertet wird. Lebensbedingungen, der von ihnen geprägte Habitus sowie die sich daraus ableitenden Erzeugungs-, Wahrnehmungs- und Bewertungsschemata (Geschmack) sind in der Summe die Beschreibung des Lebensstils eines Individuums (Bourdieu 1987). Kommer und Biermann (2012) adaptieren den bourdieuschen Habitusbegriff und beziehen ihn auf die Mediensozialisation:

,[...] ein System von dauerhaften medienbezogenen Dispositionen, die als Erzeugungs- und Ordnungsgrundlagen für mediale Praktiken und auf Medien und den Medienumgang bezogene Vorstellungen und Zuschreibungen fungieren [...].

Der mediale Habitus bezeichnet damit auch eine charakteristische Konfiguration inkorporierter, strukturierter und zugleich strukturierender Klassifikationsschemata, die für ihre Träger in der Regel nicht reflexiv werden" (Kommer und Biermann 2012, S. 90)

Die Autoren haben das Konzept des medialen Habitus empirisch in aufeinander aufbauenden Studien fundiert. Im Fokus einer Studie standen die Bildung 
medialer Habitustypen und die Zuordnung der befragten Lehramtsstudierenden zu diesen (ebenda). Als Ergebnis der Typenbildung konnten vier mediale Habitustypen ermittelt werden (verkürzte Darstellung):

1. und 2. die „,ambivalenten Bürgerlichen “ mit der Unterform der „überforderten Bürgerlichen ": [...] bildungsbürgerlich-kulturkritischer Habitus, der mit seiner Orientierung auf das ,gute Buch“ den neueren audiovisuellen Medien immer äußerst kritisch bis ablehnend gegenüberstanden hat.

3. die „,hedonistischen Pragmatiker “: [...] verfügen bereits im Elternhaus über deutlich weniger bildungsbürgerlich ausgerichtetes kulturelles Kapital als bei den „,bürgerlichen" Habitusformen. Der Umgang mit einem breiten Medienensemble wird dann auch als Normalität gelebt, wobei der Aspekt „Unterhaltung“ eine große Rolle spielt.

4. die „, kompetenten Medienaffinen “: Die selbsttätig erworbene umfangreiche Medienkompetenz ermöglicht verschiedenste Nutzungsformen eines breit gefächerten Spektrums von Inhalten. Die Medien werden ohne sichtbare Brüche sowohl zur Unterhaltung als auch zur Informationsbeschaffung und Selbstbildung genutzt (ebenda, S. 92 ff.)

Die Abb. 1 zeigt die Verortung der ermittelten medialen Habitustypen in Bezug auf die Mediensozialisationen der Befragten und deren medienbezogene Orientierung. Die meisten der befragten Lehramtsstudierenden werden dem Habitustypus „, ambivalente Bürgerliche“ mit der Unterform „,überforderte Bürgerliche“ zugeordnet. Die Autoren äußern im Zuge dieser Ergebnisse Bedenken, dass sich der mediale Habitus der Lehramtsstudierenden im späteren Schuldienst fortsetzen und diese zukünftigen Lehrkräfte abweichende Handlungsmuster ihrer Schüler*innen ablehnen werden (ebenda, S. 103).

Die Forschungsergebnisse von Kommer und Biermann sind grundlegend auf diesem Feld. Es folgen weitere, die zu einer Entwicklung des Konzeptes beitragen. Beispielsweise untersuchte Mutsch (2012) den medialen Habitus von Volksschulkindern und deren Lehrpersonen. Mutsch kann keinen eindeutigen medialen Habitustypus unter den befragten Lehrkräften herauskristallisieren, sondern ermittelt Ausprägungen von medialen Habitustypen (verkürzte Darstellung): „Unsicher-distanzierte Pragmatiker“, „kritisch-distanzierte Pragmatiker“, „souveräne Medienaffine“ sowie die „Allrounder“ (ebenda, S. 185 ff.). Weiter untersuchten ein Forschungsteam der Pädagogischen Hochschule Zürich medienbezogene Einstellungen von Lehramtsstudierenden (Scheuble et al. 2014). Ziel war es, unter anderem etwas über den medialen Habitus der Studierenden zu erfahren (ebenda, S. 8 f.). Ein ausschließlich pragmatischer Umgang mit Medien sowie eine bewahrpädagogische Haltung, wie sie beispielsweise Kommer und Biermann (2012) ermittelten, trifft auf die Mehrheit der Befragten 


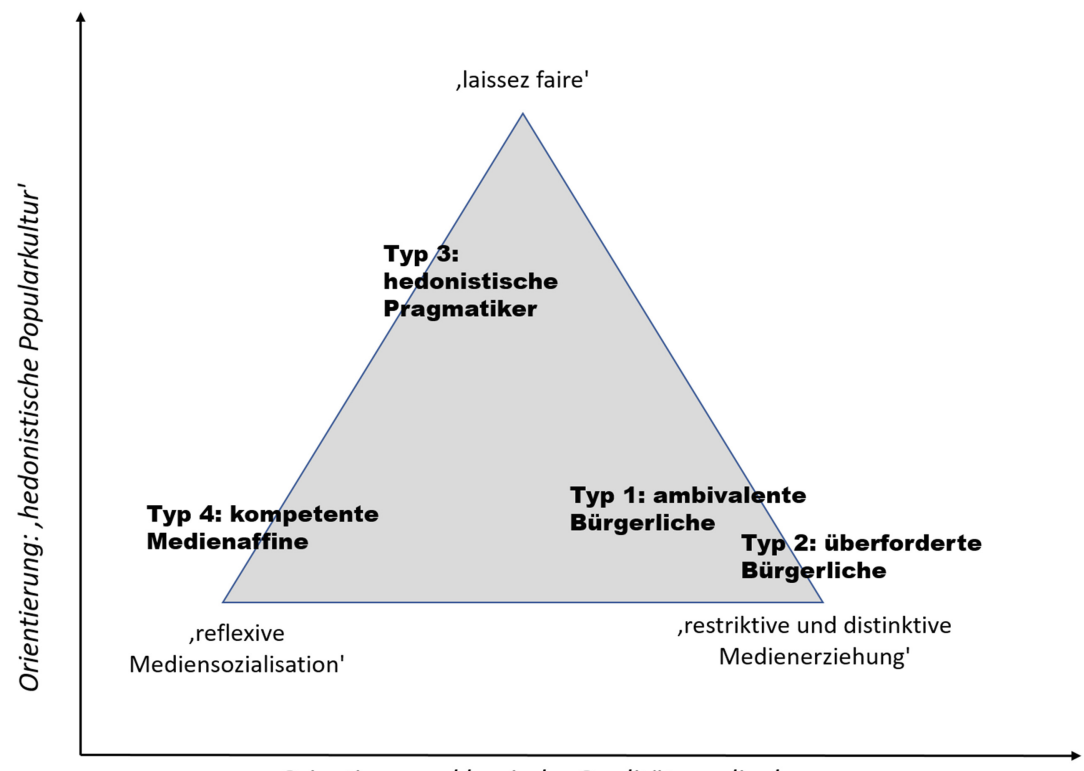

Orientierung: ,klassische Qualitätsmedien'

Abb. 1 Mediale Habitustypen bei Kommer und Biermann (2012), zwischen drei Polen verortet. (Bäsler 2019)

nicht mehr zu. Durch die Untersuchung wurde weiterhin deutlich, dass von den Studierenden eine medienbezogene Grundlagenvermittlung und medienpraktische Unterrichtskompetenzen gewünscht werden. Der Wunsch nach konkreten Einsatzszenarien im Unterricht wurde explizit (ebenda, S. 91 ff.).

Unter Bezugnahme der genannten Quellen versteht die Autorin des vorliegenden Beitrags das Konzept des medialen Habitus als eine Verkörperung medienbezogener Vorstellungen und daraus resultierenden medienbezogenen (Handlungs-)Entscheidungen, die der jeweiligen Mediensozialisation zuzuschreiben sind. 


\section{$3 \quad$ Entwicklung der Fragestellung}

\subsection{Ausgangssituation: Medienbildung in der Lehrkräftebildung an deutschen Universitäten und pädagogischen Hochschulen in Deutschland}

Die Bedeutung von Medienbildung in der Hochschulbildung wird von der Kultusministerkonferenz mehrfach betont (KMK 2012, S. 2016):

„Digitale Kompetenzen der Studierenden werden insbesondere durch die digitale Praxis in Lehre und Forschung und die Digitalisierung des Hochschulalltags gefördert“ (KMK 2016, S. 12 f.).

Um auf dem Gebiet der Medienkompetenzförderung von angehenden Lehrkräften erfolgreich zu sein, muss die Zusammenarbeit in allen Bereichen der Hochschule funktionieren (Schiefner-Rohs 2016, S. 19). Die Hochschulen müssen sich „Medienmündigkeit als Ziel von Hochschulbildung und im Prozess der Wissensund Erkenntnisgewinnung Notwendigkeit von Reflexion und Begleitung" zum Ziel setzen (ebenda, S. 26). Die Herausforderung besteht für die Hochschulen unter anderem darin, die institutionellen Rahmenbedingungen zu überarbeiten, Studierende nicht zu überfordern sowie Vernetzung und Übergänge zu gestalten (ebenda, S. 29). „Grundlage für die professionellen, medienbildnerischen Kompetenzen ist eine umfassende Medienkompetenz. Ohne das Wissen über Funktionen, Strukturen und gesellschaftliche Auswirkungen digitaler Medien, [...] Basiswissen zur Mediensozialisation von Kindern und Jugendlichen und zum Kinderund Jugendmedienschutz und nicht zuletzt auch technische Fertigkeiten, können pädagogische Fachkräfte ihren anspruchsvollen Aufgaben nicht gerecht werden“, konstatieren Büsch und Kollegen (Büsch et al. 2016, S. 8).

Eine Voraussetzung für Medienbildung an Schulen ist, dass alle Lehramtsstudierende eine medienpädagogische und mediendidaktische Grundbildung erhalten (Kammerl und Ostermann 2010). Blömeke postuliert, dass Lehrkräfte Konzepte zum Erwerb medienpädagogischer Kompetenz benötigen (Blömeke 2003, S. 5) und dass die Universität der Ort ihrer Vermittlung ist (ebenda, S. 22). Darüber, dass es eine Medienbildung für pädagogische Fachkräfte geben muss, herrscht Konsens. Über die Inhalte müsse man sich jedoch noch einig werden (Kammerl und Mayrberger 2014, S. 83 f.). 


\subsection{Medienpädagogische Grundbildung an pädagogischen Hochschulen und Universitäten}

Lorenz und Endberg (2016) fragen in einer Studie nach den Kompetenzen von Lehrkräften im medienbezogenen Bereich. Die Ergebnisse zeigen, dass sich eine überwiegende Mehrheit der bundesweit befragten Lehrkräfte in den ersten beiden Phasen der Lehrkräfteausbildung (Universität und Referendariat) in Bezug auf Medien unzureichend vorbereitet fühlt (ebenda, S. 62). In Bezug auf die Medienkompetenz der Lehrkräfte zeigt sich ein eher positives Bild. So erhob die ICILS (2013) unter anderem, dass Lehrkräfte in Deutschland und Europa Vertrauen in ihre eigenen technischen Fähigkeiten besitzen (ICILS 2013). Als problematisch wird jedoch das mangelnde Vertrauen in die eigenen medienbezogenen fachdidaktischen Kompetenzen betrachtet. Dieser Fakt könnte demnach eine Hürde für den Einsatz digitaler Medien im Unterricht darstellen (ebenda). Pietraß und Schäffer (2014) stellen die Wichtigkeit der Universität als Vermittlungsort einer Grundbildung Medien heraus, da wissenschaftliches Arbeiten ohne Mediennutzung nicht denkbar ist, und fordern daher, dass eine universitäre Grundbildung sowohl Medienbildungs- als auch Medienkompetenzkonzepte berücksichtigen sollte (ebenda, S. 101 ff.). Niesyto (2012) konstatiert: „Was in der 1. Phase der Lehrerbildung nicht grundgelegt wird, schleppt sich als Defizit in den weiteren Phasen fort" (ebenda, S. 352). Arnold et al. (2014) entwickelten und evaluierten ein Medienkompetenztraining in drei Kompetenzstufen für Lehramtsstudierende. Die Evaluation des Medienkompetenztrainings brachte unter anderem hervor, dass dieses von den teilnehmenden Lehramtsstudierenden in hohem Maße akzeptiert wurde. „Emotionale Beteiligung“ und „,inhaltliche Relevanz “ werden von der Projektgruppe als begünstigende Faktoren für den Erfolg des Medienkompetenztrainings genannt (Arnold et al. 2014).

In Hinblick auf eigene Studienergebnisse, auf die in diesem Beitrag Bezug genommen wird, wird das Moment ,inhaltliche Relevanz“ im Zuge der Medienbildung der Lehramtsstudierenden bestätigt. Lehramtsstudierende äußerten den Wunsch nach Verknüpfung von Bedienkompetenzen und Fachdidaktik (Bäsler 2019).

\subsection{Desiderata: Annahmen zum Stand der Medienbildung und Fragestellung}

Ein Ansatz für die Erklärung unzureichender Medienbildung in Schule und Unterricht kann unter anderem in der Lehrkräftebildung gesehen werden. Lehrkräfte 
seien einerseits zu schlecht in Bezug auf digitale Lehre (ICILS 2013) ausgebildet und andererseits - bedingt durch ihren medialen Habitus - gegenüber medienbezogenen Neuerungen negativ eingestellt. Orientiert an eingeführten Begriffen des medialen Habitus sowie dem Medienbildungsbegriff wurde in einer qualitativ angelegten Studie (Bäsler 2019) der Forschungsfragen nachgegangen, inwieweit sich die medienpädagogische Ausbildung von Lehrkräften und deren medialer Habitus einander bedingen (Forschungsfrage 1) und ob sich günstige und ungünstige Faktoren für die Entwicklung des medialen Habitus während der ersten Phase der Lehrkräftebildung benennen lassen (Forschungsfrage 2). Als Ergebnis der Studie wurden Implikationen für eine Verbesserung der Medienbildung und medienpädagogischen Ausbildung in der Lehrkräftebildung benannt. Zur Operationalisierung der Forschungsfragen wurden sechs Unterfragen FF1-FF6 abgeleitet:

FF1: Welche Rolle spielen Medien im Studium der Lehramtsstudierenden?

FF2: Welche Rolle spielt Medienbildung im Studium der Lehramtsstudierenden?

FF3: Wie bewerten Lehramtsstudierende das Thema Medienbildung?

FF4: Wie bewerten Lehramtsstudierende das Thema schulische Medienerziehung?

FF5: Welcher mediale Habitus lässt sich bei den Lehramtsstudierenden feststellen?

FF6: Welche medienbezogenen Vorstellungen prägen den Habitus?

Die theoretische Fundierung der sechs Unterfragen bezieht sich auf das eingangs vorgestellte Konzept des medialen Habitus sowie der Definition medienpädagogischer Kompetenz nach Blömeke (2003).

\section{$4 \quad$ Qualitatives Forschungsdesign - Methoden und Prozess der Ergebnisgewinnung}

\subsection{Datenerhebung}

Im WS 13/14 wurden $N=15$ Studierende an zwei deutschen Universitäten befragt. Die Rekrutierung der Proband*innen für die Datenerhebung fand in zwei Phasen statt: In der ersten Phase wurden Lehramtsstudierende einer Universität mit fest integriertem Medienbezug in der Lehrkräftebildung angeworben (Akronym: Medienuni). Medienbezug bedeutet in diesem Zusammenhang einen eigenen 
Fach- oder Arbeitsbereich Medien und die curriculare Verankerung des Themas Medien in der Lehrkräftebildung, welche sich im Angebot von medienbezogenen Seminaren für Lehramtsstudierende widerspiegelt. Die zehn Interviews wurden telefonisch durchgeführt und aufgezeichnet. In der zweiten Phase der Datenerhebung wurden Lehramtsstudierende einer Universität ohne curricular festgelegten Medienbezug in der Lehrkräftebildung rekrutiert (Akronym: Vergleichsuni). Insgesamt war der Rücklauf an interessierten Teilnehmer*innen geringer als an der Medienuniversität. Alle Probeinterviews und Interviews wurden in Form von Telefoninterviews von der Autorin selbst durchgeführt.

Für die Datenerhebung wurde das Instrument des halboffenen Leitfadeninterviews gewählt. In den Interviews wurden die Lehramtsstudierenden unter anderem gefragt, welche Rolle Medien in ihrem Studium spielen - sowohl inhaltlich als auch strukturell - oder welchen Stellenwert sie der schulischen Medienbildung beimessen. Damit wurde auf die Dimension der medienpädagogischen Kompetenz nach Blömeke (2003) eingegangen. Eine andere Interviewfrage zielte auf den Bildungswert ab, den die Lehramtsstudierenden verschiedenen Medien zusprechen. Damit wurde auch auf die Dimension des medialen Habitus nach Kommer und Biermann (2012) eingegangen.

Der Interviewleitfaden orientiert sich am Erkenntnisinteresse der oben genannten Fragestellungen und wurde in Probeinterviews getestet und mehrfach überarbeitet, bevor er bei der eigentlichen Stichprobe zum Einsatz kam. Nach den Probeinterviews „kristallisierten“ sich vier zentrale Fragen sowie eine These heraus, auf die die Studierenden antworten oder reagieren sollten.

1. Intervieweröffnung: Motivation zur Wahl des Lehrer*innenberufs: „Erzähl mir bitte etwas von deiner Motivation, den Lehrberuf ausüben zu wollen!“

2. Medienbildung im Studium: Bezug zu medienpädagogischer Kompetenz: „Welche Rolle spielt das Thema Medien in deinem Studium/in deiner Ausbildung?"

3. Medienpädagogische Kompetenz als Teil der Lehrkräftebildung: „Welche Rolle spielt deiner Meinung nach die Schule bei der Medienerziehung von Kindern und Jugendlichen?"

4. Medienbezogene Vorstellungen: Bezug zu medialem Habitus: „Als Nächstes möchte ich gern mit dir eine kleine Reise in deine Medienbiografie machen: Beschreibe bitte deine Mediennutzung von früher, als du noch ein Kind oder Jugendlicher warst, als du dein Studium begonnen hast, und heute, kurz bevor du ins Referendariat gehst."

5. These: Bezug zu medialem Habitus: „Lehrerinnen und Lehrer sollen Schülerinnen und Schülern in der Schule Medienkompetenz vermitteln und neue 
Medien für die Gestaltung des Unterrichts einsetzen - wie willst du das [in der Rolle der Lehrkraft] umsetzen?“

Jedes Interview wurde mit der Motivationsfrage (1) eröffnet. Die weiteren Fragen sowie zusätzliche Impulsfragen wurden flexibel gestellt, zum Beispiel „Inwiefern werden Medien in euren Seminaren thematisiert?“ Diese „Dynamik“ war erwünscht und ist charakteristisch für eine offene Herangehensweise von halboffenen Leitfadeninterviews.

\subsection{Datenanalyse}

\subsubsection{Methodisches Vorgehen}

Die Auswertung des transkribierten Audiomaterials, welches im Zuge der Datenerhebung entstanden ist, erfolgte systemisch gestützt (MAXQDA) mittels der inhaltlich-strukturierenden Qualitativen Inhaltsanalyse (im Folgenden: QIA) nach Mayring (2010). Kernstück der QIA ist die Kategorienbildung. Für die Analyse wurde die induktive Kategoriengewinnung angewendet: Die sechs Unterforschungsfragen wurden in einem ersten Materialdurchgang an das Material herangetragen und anschließend induktiv abgeleitete Hauptkategorien gebildet. In einem weiteren Materialdurchgang wurden die Hauptkategorien sodann in Subkategorien ausdifferenziert. Zur Überprüfung des so entstandenen Kategoriensystems wurden alle Interviewtexte in einem dritten Materialdurchgang den Kategorien zugeordnet. Das Kategoriensystem wurde in diesem Schritt einer Qualitätsprüfung unterzogen und noch einmal verfeinert. Um das Prinzip der Nachvollziehbarkeit zu gewährleisten, wird bei der Analyse die Reihenfolge des Ablaufmodells eingehalten. Des Weiteren schlägt die QIA die Bearbeitung des Textmaterials mithilfe eines Kodierleitfadens vor, in dem genau formuliert wird, wann Textpassagen einer Kategorie zugeordnet werden. Die einzelnen Kategorien werden dabei mit genauen Definitionen und Kodierregeln versehen (ebenda).

\subsubsection{Ergebnis der Datenanalyse: Induktiv ermitteltes Kategoriensystem}

Die Datenauswertung fand in drei Schritten statt: 1) Verschriftlichung des ermittelten Kategoriensystems mit Textbelegen, 2) Interpretation des Kategoriensystems im Sinne der Forschungsfragen und 3) Diskussion der Ergebnisse. Um die Ergebnisse der Studie vollends nachzuvollziehen, müsste das gesamte ermittelte Kategoriensystem - also alle ermittelten Kategorien und Subkategorien zu jeder der sechs Unterfragen - präsentiert werden, was den Rahmen des Beitrags 
überziehen würde. Daher wird an dieser Stelle nur ausschnittsweise auf einzelne Ergebnisse eingegangen. Für weiterführende Literatur siehe Bäsler (2019). Beispielhaft sollen hier Textpassagen aus Antworten auf zwei Forschungsfragen (FF3 und FF5) präsentiert werden. Ergänzend dazu listen Tab. 1 und 2 die ermittelten Haupt- und Subkategorien für FF3 auf.

FF3: Wie bewerten Lehramtsstudierende das Thema Medienbildung für ihre Ausbildung?

Textpassage, die der Subkategorie ,Interesse an Medieneinsatz in der Schule geweckt" zugeordnet wurde:

Interviewerin: „Und hast du denn am Ende das Gefühl gehabt, das [das Seminar] hat dir jetzt wirklich was gebracht?“

Studentin, Vergleichsuni: „Ja. Also ein wenig schon, es hat mehr mein Interesse geweckt, dass ich solche Plattformen, Lernplattformen oder Whiteboards oder so mit in meinen Unterricht integriere. Am Anfang hätte ich mich vielleicht noch eher skeptisch zu Whiteboards geäußert, jetzt würde ich denken: ,Naja, warum nicht?` Hm, dahingehend hat's ja schon was bewirkt. Und, ja, die Diskussion fand ich auch gut, also am Anfang ist es mir schwergefallen [...]. Aber letztendlich war das schon 'ne Bereicherung, fand ich.“

\section{FF5: Welcher mediale Habitus prägt die Lehramtsstudierenden?}

Textpassage, die der Subkategorie ,digitaler Pragmatiker“ zugeordnet wurde:

,[...] habe ich mich lange Zeit geweigert, mir ein internetfähiges Smartphone zu kaufen, weil ich einen Laptop habe und der Meinung war, dass ich nicht ständig erreichbar sein muss. Und um mich herum haben aber nach und nach alle mehr und

Tab. 1 Haupt- und Subkategorien der Forschungsfrage 3.

\begin{tabular}{l|l}
\hline Hauptkategorie & Mehrwert der eigenen Medienbildung \\
\hline Subkategorie & $\begin{array}{l}\text { Interesse an Medieneinsatz in der Schule } \\
\text { geweckt }\end{array}$ \\
\hline Subkategorie & $\begin{array}{l}\text { Mediennutzungskompetenz während der } \\
\text { Ausbildung erlangt }\end{array}$ \\
\hline Hauptkategorie & $\begin{array}{l}\text { Ausbildung medienpädagogischer } \\
\text { Kompetenz }\end{array}$ \\
\hline Subkategorie & $\begin{array}{l}\text { Eigene Vorstellungen von } \\
\text { Medienerziehung }\end{array}$ \\
\hline Subkategorie & $\begin{array}{l}\text { Anleitung für } \\
\text { Medienkompetenzvermittlung benötigt }\end{array}$ \\
\hline Subkategorie & Sicherheit durch Seminare erlangt \\
\hline
\end{tabular}


Tab. 2 Haupt- und Subkategorien der Forschungsfrage 3. (Eigene Darstellung)

\begin{tabular}{l|l}
\hline Hauptkategorie & $\begin{array}{l}\text { Reflexion der eigenen } \\
\text { Medienerziehung }\end{array}$ \\
\hline Subkategorie & $\begin{array}{l}\text { Viele Printmedien, wenig oder gar } \\
\text { kein Fernsehen }\end{array}$ \\
\hline Subkategorie & $\begin{array}{l}\text { Viel Unterhaltungsmedien, wenig } \\
\text { Bücher }\end{array}$ \\
\hline Subkategorie & $\begin{array}{l}\text { Liberale Medienerziehung mit wenig } \\
\text { elterlicher Kontrolle }\end{array}$ \\
\hline Subkategorie & Behütete Medienerziehung \\
\hline Hauptkategorie & $\begin{array}{l}\text { Darstellung des gegenwärtigen } \\
\text { Medienalltags }\end{array}$ \\
\hline Subkategorie & Digitaler Fan \\
\hline Subkategorie & Digitaler Pragmatiker \\
\hline Hauptkategorie & $\begin{array}{l}\text { Bewertung der eigenen } \\
\text { Medienkompetenz }\end{array}$ \\
\hline Subkategorie & $\begin{array}{l}\text { Hohe Bewertung der eigenen } \\
\text { Medienkompetenz }\end{array}$ \\
\hline Subkategorie & Durchschnittlich medienkompetent \\
\hline
\end{tabular}

mehr WhatsApp und Facebook genutzt, sodass ich mich auch gezwungen fühlte, vor einem Jahr auch ein Smartphone zu kaufen. Ich habe mich auch ganz spät bei Facebook angemeldet, aber ich kam nicht mehr drum herum, weil die Unikontakte dort auch laufen usw.“ (Studentin, Medienuni).

Die aus der Interpretation des Kategoriensystems ermittelten medialer Habitustypen „digitaler Pragmatiker“ und „digitaler Fan“ zeigen auf, dass heutige Lehramtsstudierende offen und interessiert dem Einsatz von Medien in Schule und Unterricht gegenüberstehen: Der Typ „digitaler Fan“ zeichnet sich durch ein hohes Interesse an neuen Medien aus und hat Spaß daran, sich beispielsweise mit neuen Computerprogrammen auseinanderzusetzen. Ihm bringt die Nutzung von Medien einen gewissen „Coolnessfaktor“, den er gern auslebt. Den Typen „digitaler Pragmatiker" zeichnet ein etwas späterer Kontakt zu insbesondere digitalen Medien und eine sich langsam gesteigerte Medienaffinität aus. PC und Internet hat dieser Typ zwar schon zu Schulzeiten benutzt, jedoch erst ab der Sekundarstufe II. Zudem bestand teilweise eine bewusste Ablehnung bestimmter Medien, die aufgrund wachsenden Drucks von außen nicht beibehalten werden konnte.

In der Abb. 2 werden die beiden Typen in ihrer Ausprägung in die Nähe media- 


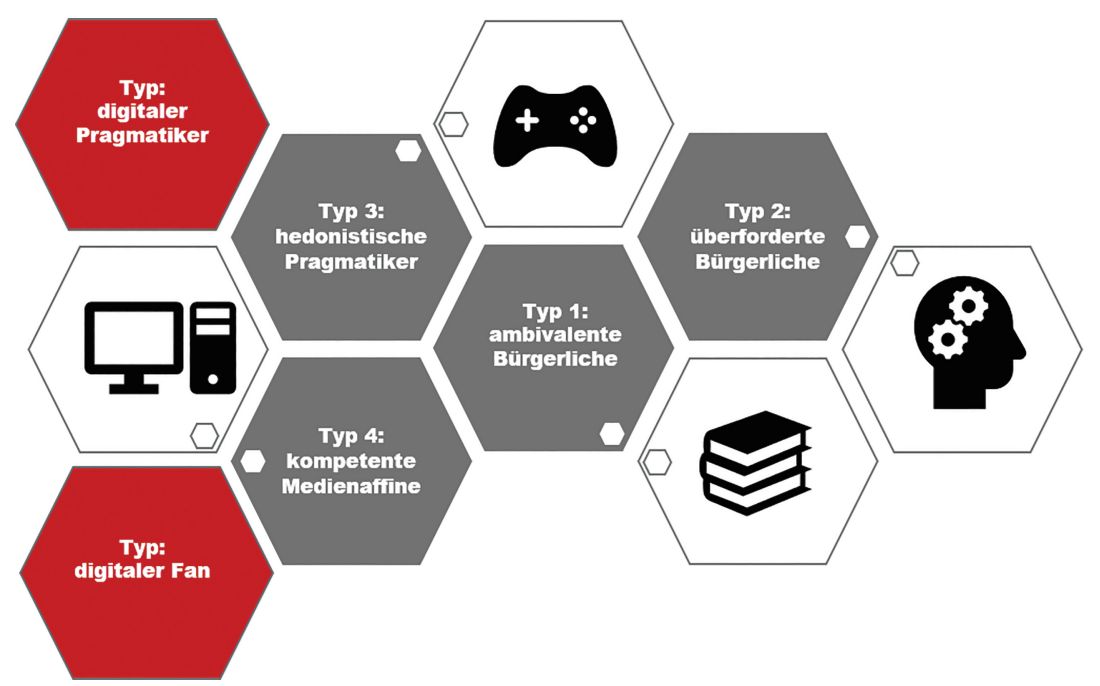

Abb. 2 Zwei mediale Habitustypen nach Bäsler (2019) neben den vier medialen Habitustypen nach Kommer und Biermann (2012). (Eigene Darstellung)

ler Habitustypen „hedonistische Pragmatiker“ und „,kompetente Medienaffine“ nach Kommer und Biermann (2012) gerückt.

\subsection{Interpretation des Kategoriensystems}

Als dritter Schritt der Analyse wurde das Kategoriensystem im Sinne der Forschungsfragen interpretiert. Durch die Bildung von thematisch passenden „Kategorienpaaren“ konnten deren Bedeutungen in Verbindung gesetzt und interpretiert werden. Beispielsweise erfolgte eine Interpretation zwischen folgenden Kategorien:

- „Entscheidung für die Schwerpunktwahl“, neue Medien während der Ausbildung.

- „gegenwärtiger Medienalltag“ der befragten Lehramtsstudierenden.

- „Entwicklung der medienbezogenen Vorstellungen“ und die damit zusammenhängenden „,Vorstellungen von Medien als Bildungsinstrumente“ der befragten Lehramtsstudierenden. 
Kurzdarstellung des Interpretationsergebnisses

Dass Studierende im Einsatz digitaler Medien für die Unterrichtsgestaltung keinen Mehrwert sehen, kann zum einen daran liegen, dass sie diesen während der Ausbildung nicht vermisst haben und ihm so auch keine Relevanz für ihre eigene Berufsausübung beimessen, und/oder zum anderen, dass sie schlechte Erfahrungen mit dem Medieneinsatz gemacht haben und ihr medialer Habitus in diesem Punkt ungünstig beeinflusst wurde. In beiden Fällen ist von einem UNGÜNSTIGEN Faktor zu sprechen. Als GÜNSTIG erweist sich das Angebot, einen curricularen Medienschwerpunkt zu wählen. GÜNSTIG ist auch die Haltung fast aller befragten Studierenden, dass allen Medien ein Bildungswert zugesprochen wird. Diese positive Grundhaltung kann nach Auswertung der Kategorien NICHT der jeweiligen Universität zugeschrieben werden.

\subsection{Ableitung: Implikationen für die Lehrkräftebildung}

Abgeleitet von den ermittelten medialen Habitustypen ,digitaler Fan“ und ,digitaler Pragmatiker" und den günstigen und ungünstigen Faktoren für die Medienbildung in der Lehrkräftebildung, ergeben sich Implikationen für die Verbesserung der Medienbildung in der Lehrkräftebildung:

- Den angehenden Lehrkräften den Mehrwert für die Nutzung von Medien in Schule und Unterricht vermitteln: Durch mangelnde Thematisierung oder einseitige Auseinandersetzung mit dem Thema neue Medien in der Lehrkräftebildung kommen Studierende zu dem Schluss, dass in dem Einsatz von Medien im Unterricht kein Mehrwert liege. Ein zu vermittelnder „Mehrwert“ liegt in den Chancen, die neue Medien beispielsweise für kollaborative Lernformate, die Anregung von Bildungsprozessen oder für den inklusiven Unterricht bieten. Eine weitere Chance liegt in der Förderung der Lernmotivation und der Medienkompetenz von Schülerinnen und Schülern (Lorenz 2018, S. 55). Ein weiterer Mehrwert liegt in der Vermittlung von Chancen und Risiken respektive im Verständnis vom Thema Künstliche Intelligenz im Bildungskontext. Die Verbindung von Künstlicher Intelligenz und digitalen Medien kann beispielsweise selbstgesteuertes und adaptives Lernen ermöglichen und fördern (Bäsler und Sasaki 2020).

- Interesse der Lehramtsstudierenden an Medienbildung wecken und fördern: Lehramtsstudierende haben Interesse am Thema neue Medien und an Medienbildung in Hinblick auf ihre Ausbildung und spätere Berufsausübung. Dieses Interesse muss von der Universität oder pädagogischen Hochschule während 
der ersten Phase der Lehrkräftebildung in Form von entsprechenden Angeboten und Lerngelegenheiten geweckt und gefördert werden - vergleiche zum Beispiel zur Akzeptanz von E-Learning und Blended-Learning-Szenarien Beckmann (2020).

- Medienbezogene Angebote gezielt für Lehramtsstudierende bereitstellen: Eine Analyse von kommentierten Vorlesungsverzeichnissen (Bäsler 2019) hat gezeigt, dass an fast allen untersuchten Universitäten und Pädagogischen Hochschulen eine Vielzahl an medienbezogenen Lehrveranstaltungen bereitgestellt wird. Dennoch mangelt es an lehramtsbezogenen Angeboten im Bereich der Medienbildung (vgl. auch Herzig und Martin 2018). Dieser Missstand wird auch in den Interviews von den befragten Lehramtsstudierenden zurückgemeldet.

- Lehramtsstudierenden Sicherheit und Selbstvertrauen im Umgang mit neuen Medien in Schule und Unterricht vermitteln. Aus den Ergebnissen der Interviewanalyse geht hervor, dass Lehramtsstudierende ein Sicherheitsbedürfnis haben, wenn es um die Erfüllung der an sie gestellten Aufgaben geht. Um ihren Aufgaben auf dem Gebiet der Medienbildung gerecht zu werden, wünschen sie sich die Vermittlung von Bedienkompetenzen auf der einen und inhaltliche Vorgaben (Handreichungen, Leitfäden) auf der anderen Seite, wenn es sowohl um den Einsatz als auch die Thematisierung von Neuen Medien in Schule und Unterricht geht. Die Förderung der eigenen Kompetenzen erhöht zudem die Motivation zur Nutzung digitaler Medien (Schütz-Pitan und Seidl und Hense 2019)

- Die medienbezogenen Vorstellungen von Lehramtsstudierenden bei der Ausgestaltung der Lehrkräftebildung berücksichtigen und einen günstigen medialen Habitus fördern: Dies kann mit einer ausgewogenen, variantenreichen, lehramtsspezifischen Medienbildung gelingen (Biermann 2013). Die Förderung der Reflexion von medialen Handlungspraktiken gehört ebenso dazu. Es geht um die Vermittlung dessen, dass die Einführung digitaler Medien in Schule und Unterricht allein nicht zwangsläufig zu einer Veränderung der Lehre führt. Lehramtsstudierende müssen ,,verstehen“, welche Potenziale in ,,anderer“ digitaler Lehre liegen. Herbeigeführt werden kann dies in einem integralen Ansatz von Medienbildung im Zuge der Lehrkräftebildung (vgl. Kerres 2020): „Nur in einer handelnden Auseinandersetzung mit digitalen Medien (immer in einem fachlichen Kontext) werden die erforderlichen Kompetenzen für Bildung in der digitalen Welt entwickelt werden können“ (ebenda, S. 16).

Dies sind nur einige Beispiele für Implikationen, weitere wurden benannt. Für weitere insbesondere theoretische Implikationen siehe Bäsler (2019). 


\section{$5 \quad$ Ausblick}

Die genannten Implikationen für die Lehrkräftebildung werden von verschiedenen Handlungsempfehlungen gestützt (zum Beispiel KMK; KBoM!; TelekomStiftung; DagstuhlSeminar; Enquetekommission der Bundesregierung; Initiative D21) ${ }^{1}$ und sind adressiert an diejenigen, die die Lehrkräftebildung an Universitäten und pädagogischen Hochschulen gestalten. Bei der Ausgestaltung der Lehre kommt den Hochschullehrenden eine große Rolle zu. Didaktische Potenziale digitaler Medien bleiben trotz vorhandener Infrastruktur an den Hochschulen häufig ungenutzt (Schmid et al. 2017). Dabei würden unter anderem Hochschulleitungen in der Digitalisierung die Lösung für konkrete Herausforderungen sehen, beispielsweise für die Umsetzung individualisierten Lernens, den Umgang mit einer heterogenen Studierendenschaft oder für das Thema Lernerfolgscontrolling. Aufseiten der Lehrenden hingegen dominiere oft noch Skepsis, was die Digitalisierung der Lehre angeht. Hochschullehrende sind letztlich dafür verantwortlich, wie oft und welche Medien Lehramtsstudierende in ihrer Ausbildung nutzen (ebenda). Die Entwicklung der technischen Möglichkeiten schreitet ungehindert fort. Seufert et al. (2020) sprechen von einer „Zweiten Welle der Digitalisierung“ und den damit verbundenen Möglichkeiten, die beispielsweise in Data Analytics oder Künstlicher Intelligenz für die Gestaltung von Lehre oder dem Managen von Hochschule liegen.

Die Entwicklung der Medienbildung in der deutschen Lehrkräftebildung ist grundsätzlich auf einem guten Weg. Insbesondere lassen die Ergebnisse der ICILS-Studie 2018 (ICILS 2018) hoffen. So geben jüngere Lehrkräfte in Deutschland zu höheren Anteilen an, dass sie im Rahmen ihrer Lehrerausbildung gelernt hätten, wie man digitale Medien im Unterricht einsetzt (ebenda). Erstrebenswert ist eine bundesländerübergreifende Standardisierung der curricularen Verankerung

\footnotetext{
${ }^{1}$ Links zu den Handlungsempfehlungen:

https://www.kmk.org/fileadmin/veroeffentlichungen_beschluesse/2012/2012_03_08_M edienbildung.pdf;

https://www.keine-bildung-ohne-medien.de/wp-content/uploads/2014/06/Stellungnahm eKMK-Papier_end.pdf;

https://www.telekom-foundation.de/dts-cms/sites/default/files/dts-library/materialien/ pdf/buch_medienbildung.bildungskette_end.pdf;

https://www.gi.de/fileadmin/redaktion/Themen/dagstuhl-erklaerung-bildung-in-der-dig italen-welt-2016.pdf;

https://www.bundestag.de/internetenquete/dokumentation/Sitzungen/20120625/A-Drs_

17_24_052_-_PG_Bildung_und_Forschung_Handlungsempfehlungen.pdf;

https://initiatived21.de/app/uploads/2017/01/d21_schule_digital2016.pdf. Zugegriffen: 11.11.2020.
} 
medienbezogener Angebote in der Lehrkräftebildung (vgl. Niesyto 2012) sowie in der Studienordnung und deren Umsetzung durch ausreichend IT-Ausstattung und Personal (vgl. Caruso und Martin 2020) als Grundvoraussetzung für vergleichbare und gleichbleibende Qualität in der Lehre sowie in Schule und Unterricht: „Für einen nachhaltigen Wandel, hin zu einer Lehrer*innenbildung, die im Stande ist, den mit der Digitalisierung einhergehenden Ansprüchen gerecht zu werden, dürfte das die entscheidende Voraussetzung sein“ (ebenda, S. 206 f.). Genauso wichtig ist es, den Studierenden selbst Gehör bei der Ausgestaltung digitaler Lehre und Medienbildung zu schenken. Studierende haben diesbezüglich konkrete Vorstellungen und Anforderungen (Baumann et al. 2019). Deren Berücksichtigung wird sich positiv auf die Akzeptanz auswirken.

\section{Literatur}

Arnold, A., Fischer, F., Franke, U., Nistor, N., \& Schultz-Pernice, F. (2014). Mediendidaktische Basisqualifikation für alle angehenden Lehrkräfte: Entwicklung und Evaluation eines Pilottrainings. https://www.pedocs.de/volltexte/2015/10891/pdf/E_Learning_2 013_Arnold_Fischer_Franke_Nistor_Schultz_Pernice_Mediendidaktische_Basisqualifi kation_fuer_angehende_Lehrkraefte.pdf. Zugegriffen: 30. Apr. 2020.

Bäsler, S.-A. (2019). Lernen und Lehren mit Medien und über Medien. Der mediale Habitus und die Ausbildung medienpädagogischer Kompetenz bei angehenden Lehrkräften. (Dissertation. Technische Universität Berlin). https://depositonce.tu-berlin.de/bitstream/ 11303/8704/4/baesler_sue_ann.pdf. Zugegriffen: 16. Apr. 2020.

Bäsler, S.-A., \& Sasaki, F. (2020). Interaktive Lernmedien. Gestaltung von digitalen Bildungsmedien mit Künstlicher Intelligenz. Information - Wissenschaft \& Praxis, 71(1), 39-42 https://doi.org/10.1515/iwp-2019-2059.

Baumann, J., Böckel, A., Denker, F., Gross, P., Kern, E., Lamprecht, M., Reimann, J., Rensinghoff, B., Sari, Z., Schopf, E., Wächtler, E., Meyer, H., Rampelt, F., \& Röwert, R. (2019): Der Digital Turn aus Studierendenperspektive. Studentisches Thesenpapier zur Digitalisierung in der Hochschulbildung. Diskussionspapier, 7. Berlin: Hochschulforum Digitalisierung. https://doi.org/10.5281/zenodo.3484574.

Beckmann, A. (2020), ,Digitalisierung in der Hochschullehre. Erfahrungen mit dem MathEdu Digital-Lehrkonzept und zur Akzeptanz digitaler Lehrelemente durch die Studierenden“. MedienPädagogik (Februar), 1-20. https://doi.org/10.21240/mpaed/00/2020.02.24.X.

Biermann, R. (2009). Der mediale Habitus von Lehramtsstudierenden. Eine quantitative Studie zum Medienhandeln angehender Lehrpersonen. Wiesbaden: Springer VS.

Biermann, R. (2013). Medienkompetenz - Medienbildung - Medialer Habitus. Genese und Transformation des medialen Habitus vor dem Hintergrund von Medienkompetenz und Medienbildung. Medienimpulse - Beiträge zur Medienpädagogik, 4. https://www.medienimpulse.at/pdf/Medienimpulse_Medienkompetenz__Medienbil dung__Medialer_Habitus_Biermann_20131203.pdf. Zugegriffen: 22. Aug. 2017. 
Blömeke, S. (2003). Neue Medien in der Lehrerausbildung. Zu angemessenen (und unangemessenen) Zielen und Inhalten des Lehramtsstudiums. MedienPädagogik: Zeitschrift für Theorie und Praxis der Medienbildung, 1-29, https://doi.org/10.21240/mpaed/00/2003. 01.11.X.

Bourdieu, P. (1987). Sozialer Sinn: Kritik der theoretischen Vernunft. Frankfurt a. M.: Suhrkamp.

Büsch, A., Knaus, T., Kommer, S., \& Missomelius, P. (2016). Stellungnahme zum Strategiepapier der Kultusministerkonferenz vom 12. Mai 2016 zu „Bildung in der digitalen Welt“. Aachen: Initiative „Keine Bildung ohne Medien!“ (KBoM!). https://www.keine-bildungohne-medien.de/stellungnahmekmkstrategiepapier/. Zugegriffen: 23. Aug. 2020.

Caruso, C., \& Martin, A. (2020). Anforderungen an Studienordnungen in einer digital vernetzten Welt. Zeitschrift für Hochschulentwicklung: Forschungsperspektiven auf Digitalisierung in Hochschulen, 15(1), 195-208.

Eickelmann, B., Aufenanger, S., \& Herzig, B. (2013). Medienbildung entlang der Bildungskette. Bonn: Deutsche Telekom Stiftung. https://www.telekom-stiftung.de/sites/def ault/files/files/media/publications/buch_medienbildung.bildungskette_end.pdf.ZZugegriffen: 23. Aug. 2017.

Herzig, B., \& Martin, A. (2018). Lehrerbildung in der digitalen Welt. In S. Ladel, J. Knopf, \& A. Weinberger (Hrsg.), Digitalisierung und Bildung (S. 89-113). Cham: Springer.

ICILS (2013). Schwippert, K., Eickelmann, B., Bos, W., Goldhammer, F., Schaumburg, H., \& Gerick, J. (2014). ICILS 2013. Münster: Waxmann.

ICILS. (2018) Eickelmann, B., Bos, W., \& Labusch, A. (2018). Die Studie ICILS 2018 im Überblick - Zentrale Ergebnisse und Entwicklungsperspektiven. In B. Eickelmann, W. Bos, J. Gerick, F. Goldhammer, H. Schaumburg, K. Schwippert, M. Senkbeil \& J. Vahrenhold (Hrsg.), ICILS 2018 \#Deutschland - Computer- und informationsbezogene Kompetenzen von Schülerinnen und Schülern im zweiten internationalen Vergleich und Kompetenzen im Bereich Computational Thinking (S. 7-31). Münster: Waxmann.

Jörissen, B., \& Marotzki, W. (2009). Medienbildung - Eine Einführung. Stuttgart: UTB $\mathrm{GmbH}$.

Kerres, M. (2020). Bildung in der digitalen Welt: Eine Positionsbestimmung für die Lehrerbildung. In M. Rothland \& S. Herrlinger (Hrsg.), Digital?! Perspektiven der Digitalisierung für den Lehrerberuf und die Lehrerbildung. Beiträge zur Lehrerbildung und Bildungsforschung. (S. 17-34) Münster: Waxmann.

Kommer, S., \& Biermann, R. (2012). Der mediale Habitus von (angehenden) LehrerInnen. Medienbezogene Dispositionen und Medienhandeln von Lehramtsstudierenden. In R. Schulz-Zander, B. Eickelmann, H. Moser, H. Niesyto, \& P. Grell (Hrsg.), Jahrbuch Medienpädagogik, 9 (S. 81-108). Wiesbaden: Springer VS.

Kammerl, R., \& Ostermann, S. (2010). Medienbildung - (k)ein Unterrichtsfach? Eine Expertise zum Stellenwert der Medienkompetenzförderung in Schulen. Norderstedt: MA $\mathrm{HSH}$.

Kammerl, R., \& Mayrberger, K. (2014). Medienpädagogik in der Lehrerbildung. Zum Status quo dreier Standorte in verschiedenen deutschen Bundesländern. In P. Imort \& H. Niesyto (Hrsg.), Grundbildung Medien in pädagogischen Studiengängen. Ansätze und Entwicklungsperspektiven (S. 81-93). München: KoPäd. 
Kultusministerkonferenz (Hrsg.) (2012). Medienbildung in der Schule (Beschluss der Kultusministerkonferenz vom 8. März 2012). https://www.kmk.org/fileadmin/veroeffentlichu ngen_beschluesse/2012/2012_03_08_Medienbildung.pdf. Zugegriffen: 30. Apr. 2020.

Kultusministerkonferenz. (2016). Strategie der Kultusministerkonferenz „Bildung in der digitalen Welt“. Beschluss der Kultusministerkonferenz vom 8.12.2016. Berlin, Bonn: Sekretariat der Ständigen Konferenz der Kultusminister der Länder der Bundesrepublik Deutschland. https://www.kmk.org/fileadmin/Dateien/veroeffentlichungen_beschluesse/ 2018/Strategie_Bildung_in_der_digitalen_Welt_idF._vom_07.12.2017.pdf. Zugegriffen: 30. Apr. 2020.

LISUM (2015). Basiscurriculum Medienbildung. Teil B Fachübergreifende Kompetenzentwicklung. Amtliche Fassung vom 10.11.2015. Berlin: Landesinstitut für Schule und Medien Berlin-Brandenburg (LISUM). https://bildungsserver.berlin-brandenburg.de/ fileadmin/bbb/unterricht/rahmenlehrplaene/Rahmenlehrplanprojekt/amtliche_Fassung/ Teil_B_2015_11_10_WEB.pdf. Zugegriffen: 30. Apr. 2020.

Lorenz, R. (2018). Ressourcen, Einstellungen und Lehrkraftbildung. In N. McElvany, F. Schwabe, W. Bos, \& H. Holtappels (Hrsg.), Digitalisierung in der schulischen Bildung. Chancen und Herausforderungen. IFS Bildungsdialoge, 2. (1. Aufl.) (S. 53-67). Münster: Waxmann.

Lorenz, R., \& Endberg, M. (2016). Digitale Medien in der Lehrerausbildung. Die Sichtweise aus der Unterrichtspraxis. merz. Medien + Erziehung. Zeitschrift für Medienpädagogik, 60(4), 60-65.

Marotzki, W. (1990). Entwurf einer strukturalen Bildungstheorie. Biographietheoretische Auslegung von Bildungsprozessen in hochkomplexen Gesellschaften. Weinheim: Deutscher Studien Verlag.

Mayrberger, K. (2012). Medienpädagogische Kompetenz im Wandel - Vorschlag zur Gestaltung des Übergangs in der Lehrerbildung am Beispiel mediendidaktischer Kompetenz. In R. Schulz-Zander, B. Eickelmann, H. Moser, H. Niesyto, \& P. Grell (Hrsg.), Jahrbuch Medienpädagogik, 9 (S. 389-412). Wiesbaden: Springer VS.

Mayring, P. (2010). Qualitative Inhaltsanalyse. Grundlagen und Techniken (11 aktualisierte). Weinheim: Beltz-Verlag.

Mutsch, U. (2012). Der mediale Habitus von Volksschulkindern und ihren Lehrerinnen und Lehrern. Eine empirische Studie zur Genese schulischer Medienkultur als Aushandlungsprozess habituellen Medienhandelns. (Dissertation, Universität Wien). https://othes.uni vie.ac.at/23971/1/2012-10-18_0104284.pdf. Zugegriffen: 19. Okt. 2020.

Niesyto, H. (2012). Bildungsprozesse unter den Bedingungen medialer Beschleunigung (2012). In G. C. Bukow (Hrsg.), Raum, Zeit, Medienbildung. Untersuchungen zu medialen Veränderungen unseres Verhältnisses zu Raum und Zeit (S. 47-66). Wiesbaden: Springer VS.

Pietraß, M., \& Schäffer, B. (2014). Grundbildung Medien an der Universität. Der Studiengang BME an der Universität der Bundeswehr München. In P. Imort \& H. Niesyto (Hrsg.), Grundbildung Medien in pädagogischen Studiengängen. Ansätze und Entwicklungsperspektiven (S. 95-107). München: KoPäd.

Scheuble, W., Signer, S., \& Moser, H. (2014). Medienbildung an der PH Zürich. Quantitative und qualitative Einschätzungen der Studierenden zur Medienbildung an der PH Zürich. Zürich: Pädagogische Hochschule Zürich. Prorektorat Ausbildung. https://media.phzh.ch/ Medium/View/14333. Zugegriffen: 30. Apr. 2020. 
Schiefner-Rohs, M. (2016). Medienkompetenz revisited - Anforderungen - Herausforderungen - Überforderungen. [Vortrag]. In E-Learning-Tag Rheinland-Pfalz. Universität Koblenz-Landau, Standort Koblenz, 27.06.2016. TU Kaiserslautern. https://2headz.ch/ blog/wp-content/uploads/2016/07/Medienkompetenz_Schiefner-Rohs.pdf. Zugegriffen: 23. Aug. 2017.

Schütz-Pitan, J., Seidl, T., \& Hense, J. (2019). Wirksamkeit eines fächer- und modulübergreifenden ePortfolio-Einsatzes in der Hochschullehre. Einflussfaktoren auf den Kompetenzerwerb. https://www.hochschullehre.org/wp-content/files/diehochschullehre_ 2019_Schuetz-Pitan_etal_Wirksamkeit_ePortfolio.pdf. Zugegriffen: 28. Apr. 2020.

Schmid, U., Goertz, L., Radomski, S., Thom, S., \& Behrens, J. (2017). Monitor Digitale Bildung: Die Hochschulen im digitalen Zeitalter. Gütersloh: Bertelsmann Stiftung. https:// doi.org/10.11586/2017014.

Seufert, S., Guggemoos, J., \& Sonderegger, S. (2020). Digitale Transformation der Hochschullehre: Augmentationsstrategien für den Einsatz von Data Analytics und Künstlicher Intelligenz. Zeitschrift für Hochschulentwicklung, 15(1), 81-101. https://doi.org/10.3217/ zfhe-15-01/05.

Spanhel, D. (2011). Medienkompetenz oder Medienbildung? Begriffliche Grundlagen für eine Theorie der Medienpädagogik. In H. Moser, P. Grell, \& H. Niesyto (Hrsg.), Medienbildung und Medienkompetenz. Beiträge zu Schlüsselbegriffen der Medienpädagogik, (neue Ausgabe) (S. 95-120). München: KoPäd.

Tulodziecki, G. (2011). Zur Entstehung und Entwicklung zentraler Begriffe bei der pädagogischen Auseinandersetzung mit Medien. In H. Moser, P. Grell, \& H. Niesyto (Hrsg.), Medienbildung und Medienkompetenz. Beiträge zu Schlüsselbegriffen der Medienpädagogik, (nеuе Ausgabe) (S. 11-40). München: KoPäd.

Open Access Dieses Kapitel wird unter der Creative Commons Namensnennung 4.0 International Lizenz (http://creativecommons.org/licenses/by/4.0/deed.de) veröffentlicht, welche die Nutzung, Vervielfältigung, Bearbeitung, Verbreitung und Wiedergabe in jeglichem Medium und Format erlaubt, sofern Sie den/die ursprünglichen Autor(en) und die Quelle ordnungsgemäß nennen, einen Link zur Creative Commons Lizenz beifügen und angeben, ob Änderungen vorgenommen wurden.

Die in diesem Kapitel enthaltenen Bilder und sonstiges Drittmaterial unterliegen ebenfalls der genannten Creative Commons Lizenz, sofern sich aus der Abbildungslegende nichts anderes ergibt. Sofern das betreffende Material nicht unter der genannten Creative Commons Lizenz steht und die betreffende Handlung nicht nach gesetzlichen Vorschriften erlaubt ist, ist für die oben aufgeführten Weiterverwendungen des Materials die Einwilligung des jeweiligen Rechteinhabers einzuholen.

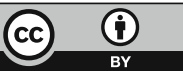

